\title{
Diabetes Mellitus, Hypercholesterolemia, and Hypertension but Not Vascular Disease Per Se Are Associated With Persistent Platelet Activation In Vivo
}

Evidence Derived From the Study of Peripheral Arterial Disease

Giovanni Davì

Paolo Gresele

Francesco Violi

Stefania Basili

Mariella Catalano

Carlo Giammarresi

Raul Volpato

Giuseppe G. Nenci

Giovanni Ciabattoni

, and

Carlo Patrono

Originally published1 Jul 1997https://doi.org/10.1161/01.CIR.96.1.69Circulation. 1997;96:69-75

\section{Abstract}

Background Previous studies relating increased thromboxane (TX) biosynthesis to cardiovascular risk factors do not answer the question whether platelet activation is merely a consequence of more prevalent atherosclerotic lesions or reflects the influence of metabolic and hemodynamic disturbances on platelet biochemistry and function.

Methods and Results We examined 64 patients with large-vessel peripheral arterial disease and 64 age- and sex-matched control subjects. TXA 2 biosynthesis was investigated in relation to cardiovascular risk factors by repeated measurements of the urinary excretion of its major enzymatic metabolite, 11-dehydro-TXB 2 , by

radioimmunoassay. Urinary 11-dehydro-TXB 2 was significantly $(P=.0001)$ higher in patients with peripheral arterial disease $(57 \pm 26 \mathrm{ng} / \mathrm{h})$ than in control subjects $(26 \pm 7 \mathrm{ng} / \mathrm{h})$. Seventy percent of patients had metabolite excretion $>2$ SD above the normal mean. However, 11dehydro- $\mathrm{TXB}_{2}$ excretion was enhanced only in association with cardiovascular risk factors. Multivariate analysis showed that diabetes, hypercholesterolemia, and hypertension were independently related to 11-dehydro- $\mathrm{TXB}_{2}$ excretion. During a median follow-up of 48 months, 8 patients experienced major vascular events. These patients had significantly 
$(P=.001)$ higher 11-dehydro- $\mathrm{TXB}_{2}$ excretion at baseline than patients who remained event free.

Conclusions The occurrence of large-vessel peripheral arterial disease per se is not a trigger of platelet activation in vivo. Rather, the rate of $T X A_{2}$ biosynthesis appears to reflect the influence of coexisting disorders such as diabetes mellitus, hypercholesterolemia, and hypertension on platelet biochemistry and function. Enhanced $\mathrm{TXA}_{2}$ biosynthesis may represent a common link between such diverse risk factors and the thrombotic complications of peripheral arterial disease.

Enhanced platelet biosynthesis of the proaggregative, vasoconstrictive substance thromboxane (TX) $A_{2}$ has been associated with several cardiovascular risk factors. These include cigarette smoking, 123 non-insulin-dependent diabetes mellitus, 4 type Ila hypercholesterolemia, $\underline{5}$ and homozygous homocystinuria. $\underline{-}$ An unresolved question is whether persistent platelet activation in these settings is merely a consequence of more prevalent atherosclerotic lesions or reflects the influence of the accompanying metabolic and hemodynamic disturbances on platelet biochemistry and function. $\underline{z}$

Arteriosclerosis obliterans of the lower limbs is an indicator of diffuse atherosclerotic disease and is associated with greatly increased cardiovascular and cerebrovascular morbidity and mortality. .9910 Moreover, a strong association of conventional risk factors with large-vessel peripheral arterial disease has been demonstrated.111213

In the present study, we sought to determine whether the biosynthesis of $\mathrm{TXA}_{2}$ is altered in vivo through repeated measurements of the urinary excretion of its major enzymatic metabolites in patients with stable, large-vessel peripheral arterial disease. We compared a group of patients without any of the major cardiovascular risk factors (diabetes, hypertension, hypercholesterolemia, or smoking) to patients with these risk factors to evaluate the relative contribution of atherosclerosis per se versus the presence of these risk factors in affecting the rate of $\mathrm{TXA}_{2}$ biosynthesis in vivo. Furthermore, we examined in a preliminary fashion the hypothesis that enhanced TXA $\mathrm{A}_{2}$ biosynthesis is associated with vascular complications during a 4-year follow-up period.

\section{Methods}

\section{Subjects}

Sixty-four patients with large-vessel peripheral arterial disease (17 women and 47 men aged $62.5 \pm 8.9$ years; range, 37 to 77 years) and 64 age- and sex-matched healthy subjects ( 17 women and 47 men aged $60.4 \pm 5.8$ years; range, 35 to 75 years) were studied at four clinical centers on several occasions between March 1989 and December 1994 (Table 1). Informed consent was obtained from each participating subject. The study protocols were approved by the internal medicine review boards of our institutions.

Patients with peripheral arterial disease were selected for having at least one of the risk factors known to be associated with enhanced TXA $\mathrm{A}_{2}$ biosynthesis, ie, cigarette smoking, diabetes mellitus, and type lla hypercholesterolemia, or none of the above. The study aimed at recruiting at least 12 patients in each group, and the length of the recruitment phase was related to the relatively rare occurrence of patients without any such risk factors. Because of the high prevalence of cigarette smoking (39\%) and hypertension $(25 \%)$ in the entire study group (Table 1), it was inevitable that such risk factors were present in a variable proportion in addition to diabetes mellitus, type lla 
hypercholesterolemia, or both. Sixty-four healthy subjects were recruited by the same participating clinical centers and matched by age and sex to the study patients. Although they were selected for not having any cardiovascular risk factors, 2 were later found to be cigarette smokers and 1 to be hypertensive.

Peripheral arterial disease was defined both by a history of intermittent claudication localized to the calf with no resting pain and relieved within 10 minutes by rest 14 and by an ankle-arm index $\leq 0.85$ at rest.

The ankle-arm index, the ratio of ankle to arm systolic blood pressure, usually $\geq 1.0$ in normal adults,, 1415161718 was measured according to a standard protocol by trained technicians. .15

The patients had unilateral $(n=12)$ or bilateral $(n=52)$ disease. In none of the patients had arterial disease undergone detectable progression during the previous 6 months as judged by clinical evaluation during outpatient visits. Moreover, all had mild-to-moderate symptoms. Approximately one third of patients had one or more additional signs of atherosclerotic arterial disease, such as stable angina pectoris, previous myocardial infarction, or a history of transient cerebral ischemia (Table 1).

Non-insulin-dependent diabetes mellitus was defined in accordance with the criteria of the American Diabetes Association.19 Type lla hypercholesterolemia was defined in accordance with $\mathrm{WHO}$ criteria ${ }^{20}$ on the basis of the determination of total plasma cholesterol and triglyceride levels after a 12-hour fast. Hypertension was defined as current systolic or diastolic blood pressure $>140 / 90 \mathrm{~mm}$ Hg. .21 Blood pressure was measured both in the supine and standing positions. Smokers were currently smoking 5 to 30 cigarettes per day. The smoking habit was confirmed by careful history documenting regular smoking for $\geq 1$ year. No participant smoked pipes or cigars. All measurements that led to categorization into the different subgroups were performed repeatedly (at least twice) during a 6-month period.

All patients were asked to abstain from taking any nonsteroidal anti-inflammatory or antiplatelet drug for at least 15 days before the study. Peripheral arterial disease patients who also had non-insulin-dependent diabetes mellitus followed an isocaloric diet and at the time of study were being treated with oral hypoglycemic drugs or insulin (intermediateacting and regular insulin) for several months. Peripheral arterial disease patients with hypercholesterolemia followed a hypocholesterolemic diet at the time of study.

Patients with liver failure or renal disease (creatinine clearance $<80 \mathrm{~mL} / \mathrm{min}$, serum creatinine level $>2 \mathrm{mg} / \mathrm{dL}$, urinary albumin excretion $>0.3 \mathrm{~g} / \mathrm{d}$ ) as well as patients with body-mass index $>28$ were excluded from the study.

\section{Design of the Studies}

In the first study, a cross-sectional comparison of 11-dehydro-TXB ${ }_{2}$ excretion was performed between patients and control subjects. Urine was collected from each subject during the 12-hour period preceding blood sampling; the samples were frozen immediately and kept at $-20^{\circ} \mathrm{C}$ until extraction. The reproducibility of $\mathrm{TXA}_{2}$ biosynthesis was assessed by obtaining an additional urine sample a week later from all patients. In 14 patients, a third urine sample was obtained after a 2-year follow-up. 
In a second study, we examined whether the metabolic disposition of $\mathrm{TXB}_{2}$ is altered in peripheral arterial disease in association with cigarette smoking 3 by measuring the urinary excretion of its major enzymatic metabolites, ie, 11-dehydro-TXB ${ }_{2}$ and 2,3-dinor$\mathrm{TXB}_{2.22}$ For this study, urine samples were obtained from six smokers with peripheral arterial disease (four men, two women; age range, 46 to 62 years) and six healthy nonsmokers (four men, two women; age range, 43 to 60 years).

A third study was designed to examine the relative contribution of platelet cyclooxygenase activity to the enhanced excretion of 11-dehydro- $\mathrm{TXB}_{2}$ associated with cardiovascular risk factors. Four patients (two with diabetes mellitus and two with hypercholesterolemia; age range, 39 to 69 years) were given aspirin (50 mg/d for 7 days), and 12-hour urine samples were obtained before and at the end of aspirin administration and on the 3rd, 5th, 7th, and 10th day after aspirin was withdrawn.

\section{Follow-up}

The vital status of the study patients was reviewed annually for 4 years to ascertain the occurrence of fatal and nonfatal vascular events. One patient died of stomach cancer and five patients were lost to follow-up because they refused to undergo the scheduled visits. These five patients were excluded from the analysis of $\mathrm{TXA}_{2}$ biosynthesis in relation to vascular complications. For this analysis, stroke, myocardial infarction, and cardiac death were considered major vascular events. Stroke was defined as rapid onset of a neurological deficit that persisted for $\geq 24$ hours unless death supervened and included specific localizing findings confirmed by neurological examination or brain scan, with no evidence of an underlying nonvascular cause. Determination of fatal stroke was based on death certificate data plus data on preterminal hospitalization with a definite diagnosis of stroke. Nonfatal myocardial infarction was defined as typical symptoms plus either typical ECG changes (including new $Q$ waves) or significant enzyme elevation. Determination of fatal myocardial infarction was based on death within 4 weeks after myocardial infarction. Cardiac death was defined as death within 24 hours of the onset of severe cardiac symptoms, unrelated to other known causes.

\section{Analyses}

Immunoreactive 11-dehydro- $\mathrm{TXB}_{2}$ and 2,3-dinor- $\mathrm{TXB}_{2}$ were extracted from 20-mL urine aliquots and analyzed by previously validated radioimmunoassay techniques. ${ }^{2324}$

All blood samples for lipid, lipoprotein, and apolipoprotein analyses were drawn into sodium and potassium EDTA (1 mg/mL). Cholesterol and triglycerides were determined enzymatically. HDL cholesterol was determined by the phosphotungstic acid/ $\mathrm{MgCl}_{2}$ precipitation method. LDL cholesterol was calculated by Friedewald's formula. .25 These procedures have been described in detail elsewhere. $\underline{26}$

\section{Statistical Analysis}

Statistical analysis was performed by use of $X^{2}$ statistics or Fisher's exact test (if $\left.n \leq 5\right)$ for independence and by unpaired $t$ test. The linear regression test was used to assess the correlation between continuous variables. When necessary, log transformation was used to normalize the data or appropriate nonparametric tests were used. .7 The aspirin study was analyzed with the Kruskal-Wallis method and Mann-Whitney $U$ test adjusted for multiple comparisons. Moreover, the association of 11-dehydro-TXB $\mathrm{T}_{2}$ excretion with the different cardiovascular risk factors was assessed by multiple regression analysis. 


\section{Results}

The rate of $\mathrm{TXA}_{2}$ biosynthesis, as reflected by the excretion of its major enzymatic metabolite, 11-dehydro-TXB ${ }_{2}$, was significantly $(P=.0001)$ higher in patients with peripheral arterial disease $(57 \pm 26$ and $60 \pm 32 \mathrm{ng} / \mathrm{h}$, as assessed on two different occasions) than in age- and sex-matched control subjects $(26 \pm 7 \mathrm{ng} / \mathrm{h})$. In $45(70 \%)$ of the 64 patients, metabolite excretion was $>2$ SD above the control mean (Fig 1).

\section{Influence of Risk Factors}

We analyzed 11-dehydro-TXB ${ }_{2}$ excretion in prospectively selected subgroups of patients with peripheral arterial disease based on the presence of cardiovascular risk factors previously associated with enhanced $\mathrm{TXA}_{2}$ biosynthesis. $\frac{123456}{}$ As detailed in Table 2, 11dehydro- $\mathrm{TXB}_{2}$ excretion was significantly higher in patients with peripheral arterial disease in association with cigarette smoking, non-insulin-dependent diabetes mellitus, hypercholesterolemia, or both diabetes mellitus and hypercholesterolemia than in control subjects adequately matched for age and sex. Despite comparable arterial disease (Table 3 ), patients who had none of the above risk factors excreted 11-dehydro- $\mathrm{TXB}_{2}$ at a rate indistinguishable from that of control subjects. Moreover, there was no statistically significant correlation between metabolite excretion and the ankle-arm index $(r=.09 ; P=.4742)$.

As shown in Fig 2, only 1 (7\%) of the 14 patients with peripheral arterial disease but without risk factors had 11-dehydro- $\mathrm{TXB}_{2}$ excretion above the normal range, in contrast to $67 \%$ to $83 \%$ of the other subgroups. Hypertensive patients excreted 11 -dehydro- $\mathrm{TXB}_{2}$ at a nonsignificantly $(P=.1267)$ higher rate than normotensive patients: 75 (median; range 23 to $123 \mathrm{ng} / \mathrm{h}$ ) versus $50 \mathrm{ng} / \mathrm{h}$ (range, 20 to $111 \mathrm{ng} / \mathrm{h}$ ). Because of the high prevalence of cigarette smoking (30\% to $42 \%$ ) and hypertension (25\% to $38 \%$ ) in the subgroups of patients with other risk factors, we performed a multiple regression analysis of 11-dehydro$\mathrm{TXB}_{2}$ excretion rates. Such analysis revealed that only diabetes mellitus (regression coefficient of 14.0 ; standard error of $6.1 ; P<.03$ ), hypercholesterolemia (regression coefficient of 22.4 ; standard error of $6.3 ; P<.001$ ), and hypertension (regression coefficient of 14.1 ; standard error of $7.1 ; P<.05)$ were independently related to 11 -dehydro$\mathrm{TXB}_{2}$ excretion.

\section{Reproducibility of TX Biosynthesis}

In 14 nonselected patients, including patients with $(n=11)$ and without $(n=3)$ cardiovascular risk factors, urinary 11-dehydro- $\mathrm{TXB}_{2}$ excretion was measured again after a 2-year followup. Metabolite excretion at 2 years averaged $52 \pm 21$ versus $52 \pm 20$ and $59 \pm 29 \mathrm{ng} / \mathrm{h}$ in the two samples obtained at baseline. The intrasubject coefficient of variation of 11-dehydro$\mathrm{TXB}_{2}$ excretion averaged $23 \pm 7 \%$ on the basis of three metabolite measurements in each patient. The 3 patients without risk factors, who had perfectly normal metabolite excretion rates at baseline $(28 \pm 8 \mathrm{ng} / \mathrm{h})$, continued to have normal values at 2 years $(28 \pm 2 \mathrm{ng} / \mathrm{h})$ with an intrasubject coefficient of variation $(22 \pm 4 \%)$ indistinguishable from that of patients with risk factors $(23 \pm 8 \%)$.

\section{Does Cigarette Smoking Alter TX Metabolism?}

Cigarette smoking has been reported to alter $\mathrm{TXB}_{2}$ metabolism in humans. $\frac{3}{\mathrm{~T}}$ Thus, we performed paired measurements of 11-dehydro-TXB ${ }_{2}$ and 2,3-dinor- $\mathrm{TXB}_{2}$, the major enzymatic metabolites of $\mathrm{TXB}_{2}$ originating via the 11-hydroxy-dehydrogenase and $\beta$ oxidation pathways, respectively, in six patients with peripheral arterial disease who were 
current cigarette smokers and in six age- and sex-matched healthy nonsmokers. The urinary excretion of 11-dehydro- $\mathrm{TXB}_{2}$ was enhanced in patients versus control subjects (59 \pm 19 versus $28 \pm 6 \mathrm{ng} / \mathrm{h} ; P=.0037)$ to the same extent as that of 2,3 -dinor-TXB ${ }_{2}(39 \pm 17$ versus $17 \pm 6 \mathrm{ng} / \mathrm{h} ; P=.0262)$, thus resulting in a comparable ratio between the two metabolites of 1.5 versus 1.6 in patients and control subjects, respectively. A highly significant linear correlation was found between individual excretion rates of the two $\mathrm{TXB}_{2}$ metabolites (Fig 3).

\section{Effects of Low-Dose Aspirin on TX Biosynthesis}

To characterize the platelet dependence of enhanced TXA $\mathrm{T}_{2}$ biosynthesis in patients with peripheral arterial disease and cardiovascular risk factors, we assessed the extent of suppression and pattern of recovery of 11-dehydro-TXB ${ }_{2}$ excretion in response to low-dose aspirin (50 mg/d for 7 days). We studied four patients with non-insulin-dependent diabetes mellitus or hypercholesterolemia. The basal rate of 11-dehydro- $\mathrm{TXB}_{2}$ excretion averaged $47 \pm 9 \mathrm{ng} / \mathrm{h}$ and was significantly $(P=.0011)$ reduced by $\approx 75 \%$, well into the normal range, at the end of 1 week of aspirin administration. As shown in Fig 4, the pattern of recovery of 11-dehydro- $\mathrm{TXB}_{2}$ excretion after aspirin withdrawal was linear over the next 10 days, a finding consistent with the slow pattern of recovery of platelet cyclooxygenase activity after acetylation by aspirin. $\underline{28}$

\section{TX Biosynthesis in Relation to Vascular Complications}

During a median follow-up of 48 months, eight patients experienced major vascular events. There were four acute myocardial infarctions (three nonfatal and one fatal), three acute ischemic strokes (one nonfatal and two fatal), and one cardiac death. Patients who experienced these events during follow-up had significantly $(P=.001)$ higher 11-dehydro$\mathrm{TXB}_{2}$ excretion at baseline than patients who remained event free: 91 (range, 67 to 127 $\mathrm{ng} / \mathrm{h}$ ) versus $50 \mathrm{ng} / \mathrm{h}$ (range, 16 to $124 \mathrm{ng} / \mathrm{h}$ ). Eight (100\%) of eight and $21(41 \%)$ of 51 , respectively, had metabolite excretion in excess of the median value (56 $\mathrm{ng} / \mathrm{h})$.

\section{Discussion}

We have previously reported 456 that the rate of $\mathrm{TXA}_{2}$ biosynthesis is persistently increased in patients with metabolic disorders associated with enhanced risk of thrombotic complications. These include non-insulin-dependent diabetes mellitus, 4 type Ila hypercholesterolemia, $\underline{5}$ and homozygous homocystinuria. $\underline{6}$ Abnormally high 11-dehydro$\mathrm{TXB}_{2}$ excretion could be largely suppressed by low-dose aspirin in these studies, thus suggesting that it reflected a platelet source of $\mathrm{TXA}_{2}$ biosynthesis. Moreover, pharmacological intervention with intensive insulin treatment, 4 simvastatin, $\underline{26}$ or probucol $\underline{6}$ allowed us to demonstrate a role for glycemic control, blood cholesterol levels, and oxidative mechanisms, respectively, as determinants of platelet activation in these clinical settings. However, because these complex metabolic disorders are also accompanied by accelerated atherosclerosis and more prevalent vascular lesions, it was not possible in these studies to assess the contribution of vascular disease vis-à-vis the underlying metabolic disorder in determining a state of persistent platelet activation.

The present study was designed to investigate the relative importance of diffuse atherosclerotic lesions and cardiovascular risk factors in affecting the determinants of platelet activation. Therefore, we investigated the rate of $\mathrm{TXA}_{2}$ biosynthesis 2930 in a relatively large group of patients with peripheral arterial disease, carefully characterized in terms of the extent of vascular involvement and presence of cardiovascular risk factors previously associated with enhanced $\mathrm{TXA}_{2}$ biosynthesis. 
The main finding of the present study is that large-vessel peripheral arterial disease per se is not a trigger of platelet activation in vivo. Rather, the rate of $\mathrm{TXA}_{2}$ biosynthesis appears to reflect the influence of diabetes mellitus, hypercholesterolemia, and hypertension on platelet biochemistry and function. We used the ankle-arm blood pressure index as a measure of the extent of vascular involvement 111617 and compared subgroups of patients with presumably comparable severity of atherosclerotic disease.

In a prospective population study in Sweden, 1 an ankle-arm index $<0.9$ was found to be a more powerful marker of generalized arteriosclerotic disease than an ultrasonographically detected carotid stenosis of $\geq 30 \%$. All of our patients had an ankle-arm index $<0.85$, with mean values ranging between 0.60 and 0.63 in the five different subgroups examined (Table 3). In the subgroup of patients without major cardiovascular risk factors, mean 11dehydro-TXB ${ }_{2}$ excretion was within $1 \mathrm{SD}$ of the control mean value in age- and sexmatched healthy subjects (Table 2). Moreover, in the entire study population, there was no relationship between the ankle-arm index and the rate of 11-dehydro- $T \mathrm{XB}_{2}$ excretion, thus suggesting that the presence of diffuse vascular lesions in patients with large-vessel peripheral arterial disease does not provide a stronger stimulus to platelet activation than that provided by a lesser degree of vascular involvement in healthy control subjects of comparable age. Similarly, $\mathrm{TXA}_{2}$ biosynthesis was normal in the patients with stable coronary disease studied by Fitzgerald et al. .22

In contrast, the vast majority (ie, $70 \%$ to $80 \%$ ) of patients with coexisting risk factors had abnormally high $\mathrm{TXA}_{2}$ biosynthesis that was reproducible over an extended period of observation. On the basis of multiple regression analysis, diabetes mellitus, hypercholesterolemia, and high blood pressure were independently related to 11-dehydro$\mathrm{TXB}_{2}$ excretion. Cigarette smoking, though associated with enhanced $\mathrm{TXA}_{2}$ biosynthesis as reported previously in subjects without peripheral vascular disease, $\underline{123}$ was not independently correlated with metabolite excretion. We examined the possibility of underestimating the actual rate of TXA $\mathrm{T}_{2}$ biosynthesis in cigarette smokers because of reduced conversion of $T X B_{2}$ to 11-dehydro- $T X B_{2}$, as described in healthy cigarette smokers. ${ }^{3}$ However, paired measurements of 11-dehydro- $\mathrm{TXB}_{2}$ and of 2,3-dinor-TX政, a major product of $\beta$-oxidation, 18 revealed a remarkably similar ratio between the two in patients and control subjects (Fig 3). These results tend to exclude altered metabolic disposition of $\mathrm{TXB}_{2}$ as a result of cigarette smoking, consistent with data of Rangemark et al. $\underline{3}$

The independent contribution of high blood pressure in affecting the rate of $\mathrm{TXA}_{2}$ biosynthesis is an unexpected finding of the present study in light of the negative findings in a previous study of patients with mild essential hypertension. ${ }^{34}$ In contrast, the independent role of diabetes mellitus and hypercholesterolemia as major determinants of enhanced $\mathrm{TXA}_{2}$ biosynthesis in the setting of peripheral arterial disease confirms earlier findings in patients with non-insulin-dependent diabetes mellitus 4 and type Ila hypercholesterolemia. $\underline{5}$

Enhanced $\mathrm{TXA}_{2}$ biosynthesis detected in association with diabetes mellitus and hypercholesterolemia was largely suppressed by a daily regimen of low-dose aspirin, and recovery of 11-dehydro- $\mathrm{TXB}_{2}$ excretion showed a time course that reflected the rate of platelet turnover (Fig 4). Although extraplatelet sources might contribute to total body synthesis of $\mathrm{TXA}_{2}$, such contribution appears to be small in patients with peripheral arterial disease and comparable to that previously established in healthy subjects. .35 
Previous studies 363738399041424344454647 examined various aspects of platelet function, including $\mathrm{TXA}_{2}$ biosynthesis, in patients with peripheral arterial disease. A large proportion of these studies reported abnormal platelet function, as measured ex vivo by various techniques, ${ }^{, 04142}$ or detected high circulating levels of platelet products. ${ }^{4344554647}$ The limitations of these capacity indexes as well as the pitfalls of plasma measurements of platelet products have been discussed previously. $\underline{70}$

The noninvasive measurement of 11-dehydro- $\mathrm{TXB}_{2}$ excretion, a widely accepted method of assessing platelet function in vivo,,${ }^{730}$ has been used in very few studies of patients with peripheral arterial disease. .63773839 However, these studies were both too small and possibly confounded by the uncharacterized presence of cardiovascular risk factors to provide a reliable assessment of the contribution of the latter vis-à-vis the underlying vascular disease in determining the rate of $T X A_{2}$ biosynthesis.

The limited follow-up data from the present study suggest that enhanced $\mathrm{TXA}_{2}$ biosynthesis and TXA $\mathrm{A}_{2}$-mediated amplification of platelet activation in response to plaque fissuring may represent a common link between different risk factors (namely, diabetes mellitus, hypercholesterolemia, and hypertension) and the occurrence of thrombotic complications in patients with peripheral arterial disease.

Despite an obvious rationale, there is still substantial uncertainty as to the clinical indication for antiplatelet therapy in patients with peripheral arterial disease because of inadequate trials in this setting. 48 The Antiplatelet Trialists' Collaboration identified more than 20 randomized trials of antiplatelet drugs in more than 3000 patients with intermittent claudication and described a $20 \%$ odds reduction of major vascular events, which failed to reach statistical significance. $\underline{48}$ Additional trials 49 have not resolved such statistical uncertainty about the efficacy of antiplatelet drugs in these patients. It is interesting to note that aspirin was used in only 5 of 27 antiplatelet trials, at doses ranging between 975 and $1500 \mathrm{mg} / \mathrm{d}$. 48 Thus, it might be argued that the proper antiplatelet regimen, ie, aspirin 75 to $100 \mathrm{mg} / \mathrm{d}, 50$ has not been tested in patients with peripheral arterial disease. Clearly, a trial of adequate size is needed to assess the efficacy and safety of low-dose aspirin in this setting. The results of the present study may help to identify a group of patients ideally suited for such a trial, ie, those with enhanced 11-dehydro-TXB 2 excretion. Moreover, this approach may help define guidelines for antiplatelet therapy in patients with peripheral arterial disease.

Table 1. Baseline Characteristics of Patients With Peripheral Arterial Disease and Age- and Sex-Ma Subjects

Characteristic

Age, y

Sex (male/female)

Ankle/arm index
Patients $(\mathrm{n}=64)$

$62.4 \pm 8.9$

$47 / 17$

$0.60 \pm 0.1$
Control Subjects

$60.4 \pm 5.8$

$47 / 17$

$0.96 \pm 0.1$ 
Table 1. Baseline Characteristics of Patients With Peripheral Arterial Disease and Age- and Sex-Ma Subjects

Characteristic

Regular cigarette smoking, $\mathrm{n}$

Hypertension, $n$

Type II diabetes mellitus, $\mathrm{n}$

Clinical evidence of stable coronary or cerebrovascular disease, $\mathrm{n}$

Plasma cholesterol, $\mathrm{mg} / \mathrm{dL}$

Plasma triglycerides, $\mathrm{mg} / \mathrm{dL}$

Concomitant medication, $\mathrm{n}$

None

Antiarrhythmics

Digitalis

Antianginals

Antidiabetics

Diuretics

Antihypertensives

Values are mean $\pm S D$.
Patients $(n=64) \quad$ Control Subjects

25

2

16

1

26

0

21

0

$216 \pm 51$

$179 \pm 25$

$155 \pm 96$

$129 \pm 33$

23

64

2

2

10

26

5

13 
Table 2. Urinary Excretion Rate (ng/h) of 11-Dehydro-Thromboxane $B_{2}$ in Subgroups of Peripheral $A$ Patients With or Without Major Cardiovascular Risk Factors Compared With Age- and Sex-Matched

Risk Factor

None $(n=14)$

Cigarette smoking $(n=12)$

Diabetes mellitus $(\mathrm{n}=16)$

Hypercholesterolemia $(\mathrm{n}=12)$

Hypercholesterolemia and diabetes $(n=10)$

Values are mean $\pm S D$.
Patients

$32.0 \pm 18.0$

$49.7 \pm 16.9$

$67.0 \pm 29.0$

$72.7 \pm 15.5$

$77.2 \pm 28.8$
Control Subjects

$27.2 \pm 7.3$

$27.3 \pm 5.7$

$28.2 \pm 7.9$

$24.7 \pm 8.9$

$24.5 \pm 7.1$

Table 3. Ankle-Arm Index in Subgroups of Peripheral Arterial Disease Patients With or Without Majo Risk Factors Compared With Age- and Sex-Matched Healthy Subjects

Risk Factor

None $(n=14)$

Cigarette smoking $(\mathrm{n}=12)$

Diabetes mellitus $(\mathrm{n}=16)$

Hypercholesterolemia $(\mathrm{n}=12)$

Hypercholesterolemia and diabetes $(n=10)$
Patients

$0.63 \pm 0.07$

$0.60 \pm 0.07$

$0.61 \pm 0.10$

$0.62 \pm 0.12$

$0.60 \pm 0.12$

\section{Control Subjects}

$0.95 \pm 0.05$

$0.97 \pm 0.06$

$0.95 \pm 0.06$

$0.97 \pm 0.08$

$0.97 \pm 0.06$

Values are mean $\pm S D$. 


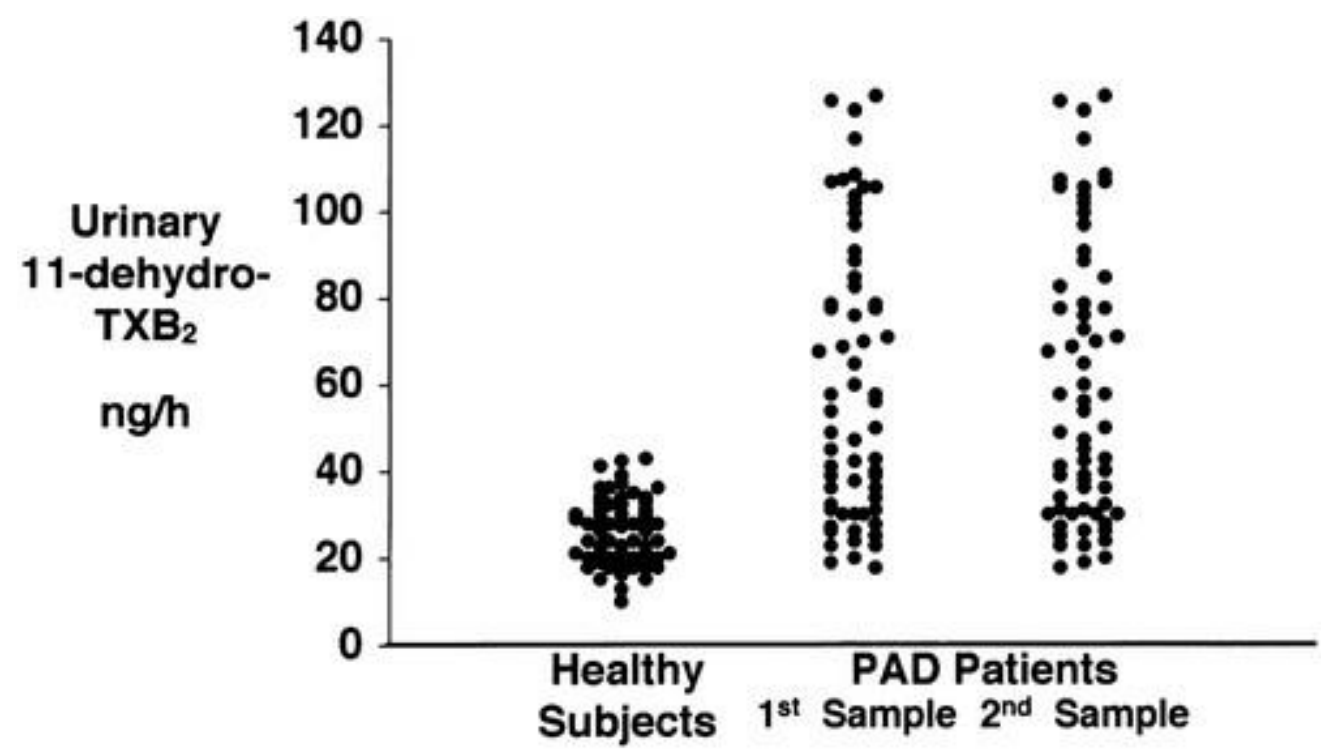

- Download figure

- Download PowerPoint

Figure 1. Urinary excretion rates of 11-dehydro-thromboxane $B_{2}\left(T X B_{2}\right)$ in 64 patients with peripheral arterial disease (PAD) and 64 sex- and age-matched healthy subjects. Dots represent individual measurements. Individual patient data are also represented in a second sample obtained 1 week later.

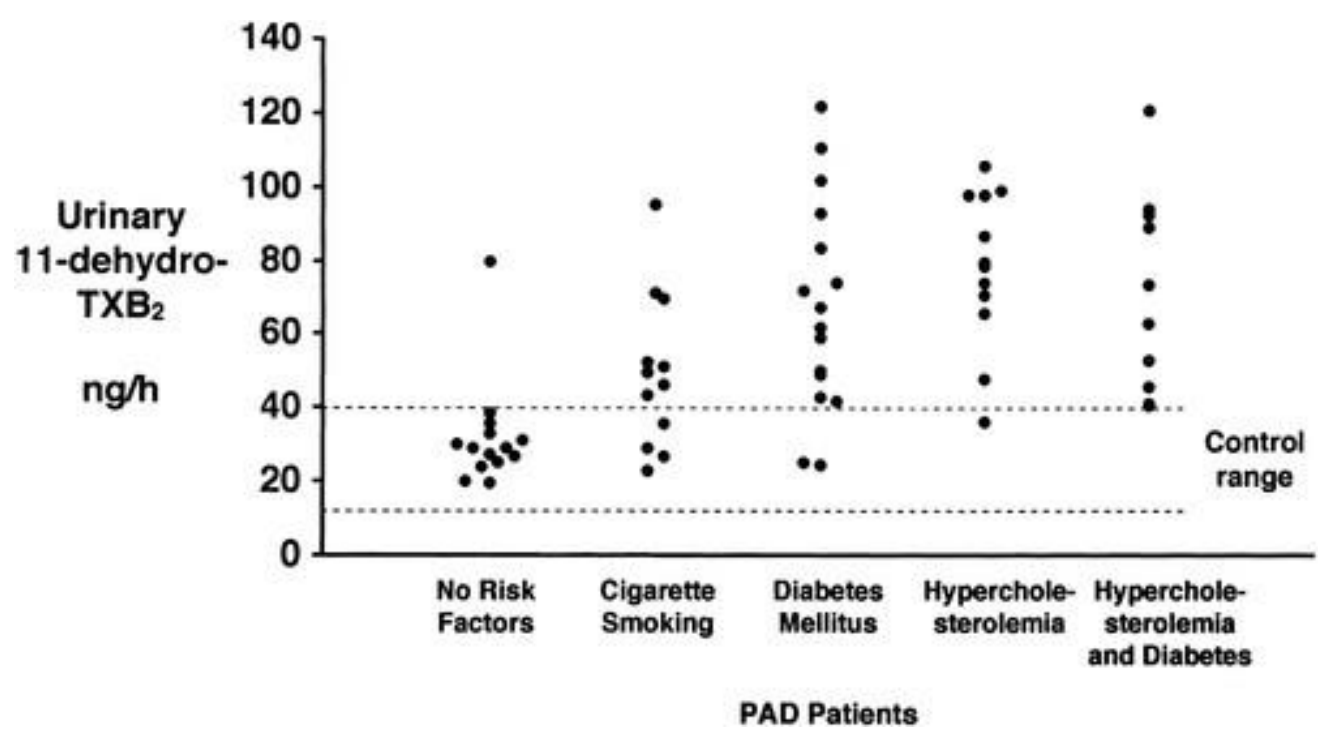

- Download figure

- Download PowerPoint

Figure 2. Urinary excretion rates of 11-dehydro- thromboxane $B_{2}\left(T_{X} B_{2}\right)$ in subgroups of patients with peripheral arterial disease (PAD). These patients were prospectively selected for having at least one of the risk factors known to be associated with enhanced $\mathrm{TXA}_{2}$ biosynthesis, ie, cigarette smoking, diabetes mellitus, and type lla hypercholesterolemia, or none of the above. The dotted lines represent 2 SD above and below the mean excretion rate in healthy control subjects. 


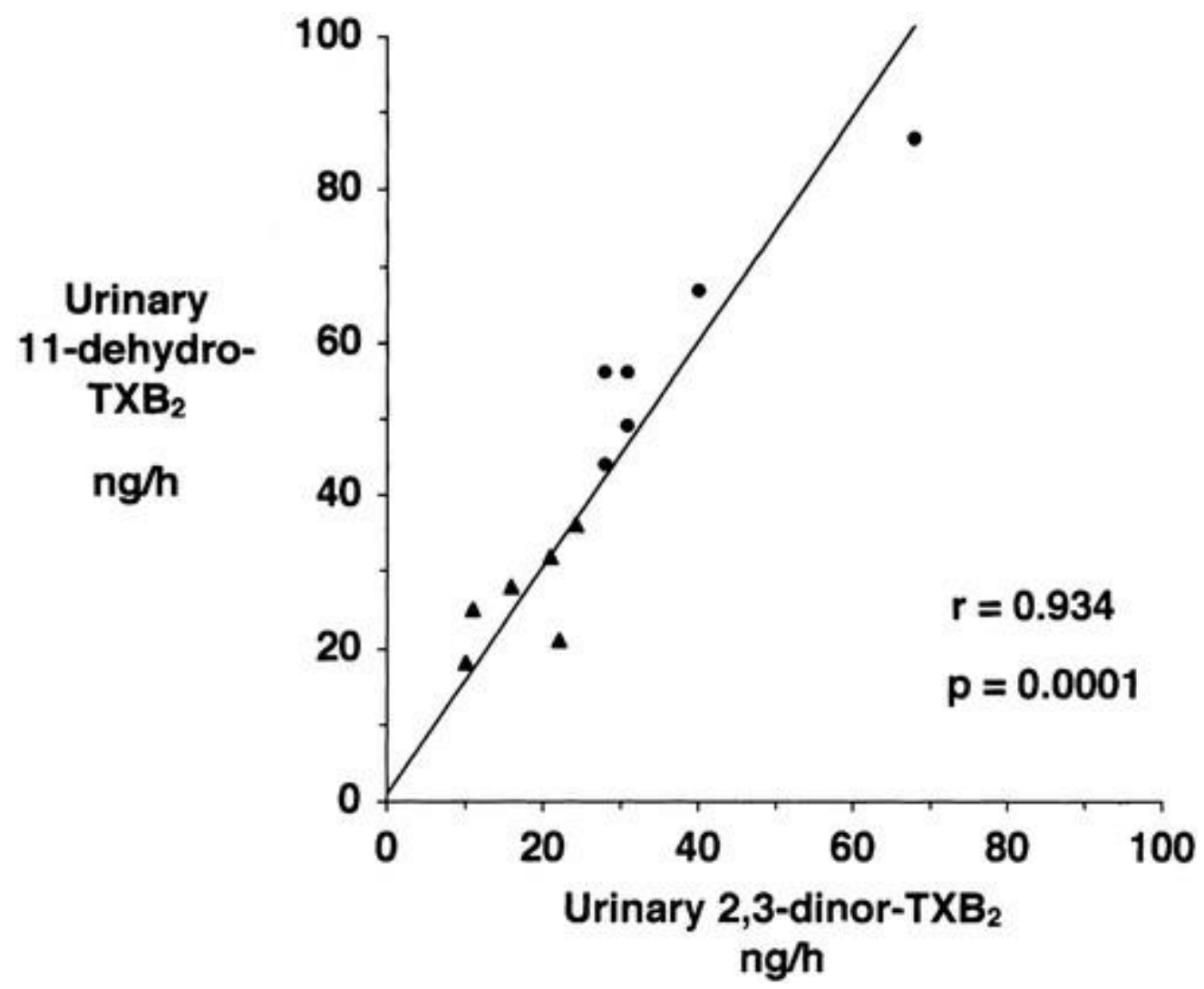

- Download figure

- Download PowerPoint

Figure 3. Correlation between the urinary excretion rates of 11-dehydro-thromboxane $\mathrm{B}_{2}\left(\mathrm{TXB}_{2}\right)$ and of 2,3-dinor-TXB in six patients with peripheral arterial disease who were current cigarette smokers $(\cdot)$ and in six age- and sex-matched control subjects $(\bullet)$ who were nonsmokers. Linear regression analysis yielded a statistically significant correlation $(r=.934 ; P=.0001)$ between the two metabolites.

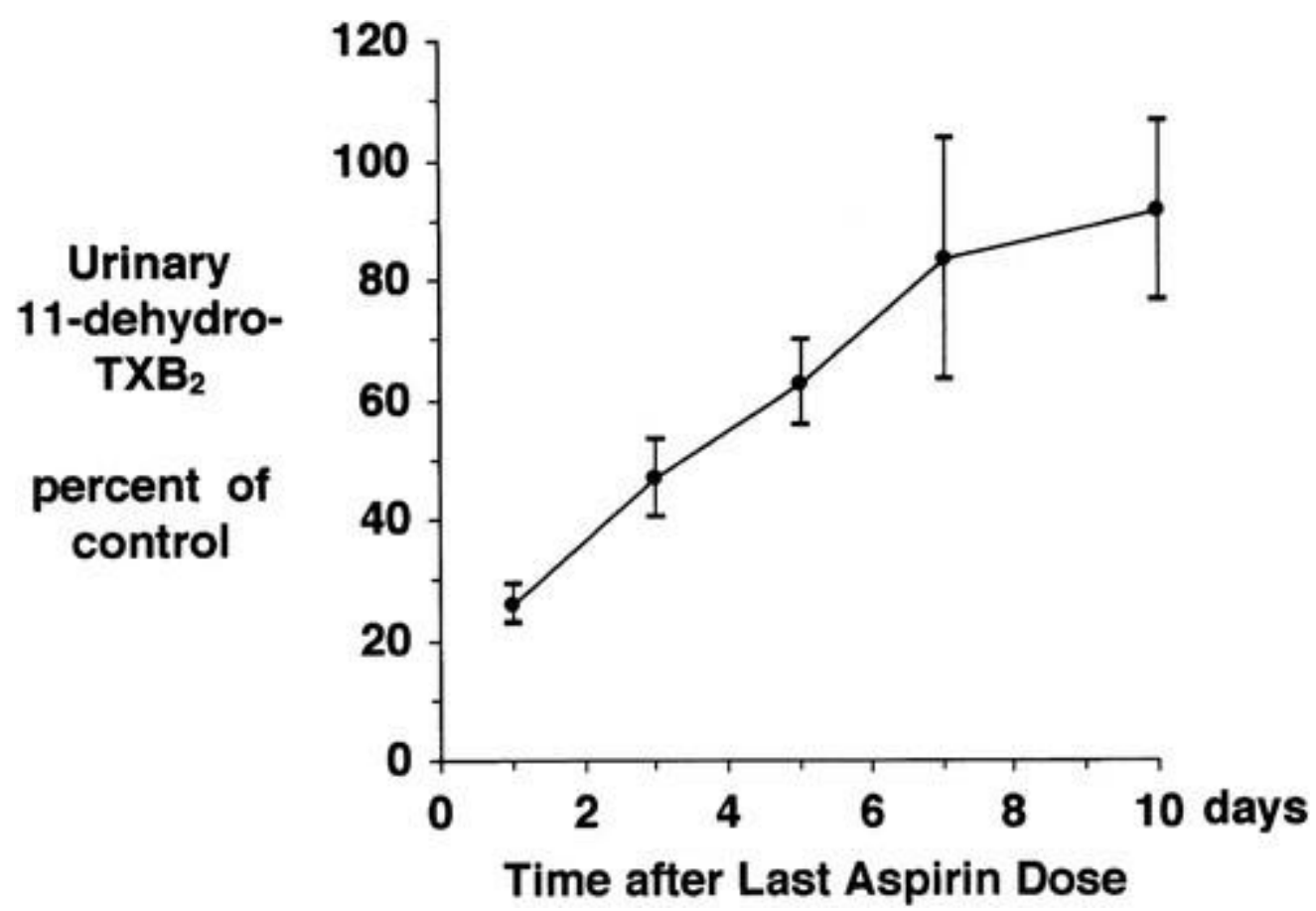


- Download figure

- Download PowerPoint

Figure 4. Urinary excretion of 11-dehydro-thromboxane $B_{2}\left(T X B_{2}\right)$ in response to administration of aspirin in low doses (50 mg/d) for 1 week to four patients with peripheral arterial disease with non-insulin-dependent diabetes mellitus or hypercholesterolemia. The graph shows the slow recovery of metabolite excretion after aspirin withdrawal. The level of 11-dehydro- $\mathrm{TXB}_{2}$ is expressed as a percentage (mean $\pm \mathrm{SD}$ ) of the level observed before aspirin administration. Each patient served as his or her own control.

This study was supported by grants from Consiglio Nazionale delle Ricerche (CNR), Progetto Finalizzato Prevenzione e Controllo dei Fattori di Malattia (SP8: 94.00560.PF41, 94.00627.PF41, 95.00882.PF41, and 95.00807.PF41). We are indebted to Dr Rino Migliacci for patient referral and helpful criticism and to Rossella Tonelli, Alessandra Migliavacca, and Andre Harris for expert editorial assistance.

\section{Footnotes}

Correspondence to Prof Carlo Patrono, Cattedra di Farmacologia I, Università degli Studi "G. D’Annunzio," Via dei Vestini, 31, 66013 Chieti, Italy. E-mail cpatrono@unich.it

\section{References}

- 1 Nowak J, Murray JJ, Oates JA, FitzGerald GA. Biochemical evidence of a chronic abnormality in platelet and arterial function in healthy individuals who smoke cigarettes. Circulation.1987; 76:6-14.CrossrefMedlineGoogle Scholar

- 2Barrow SE, Ward PS, Sleightholm MA, Ritter JM, Dollery CT. Cigarette smoking: profiles of thromboxane- and prostacyclin-derived products in human urine. Biochim Biophys Acta.1989; 993:121-127.CrossrefMedlineGoogle Scholar

- 3Uedelhoven WM, Rutzel A, Meese CO, Weber PC. Smoking alters thromboxane metabolism in man. Biochim Biophys Acta.1991; 108:197-201.Google Scholar

- 4Davì G, Catalano I, Averna M, Notarbartolo A, Strano A, Ciabattoni G, Patrono C. Thromboxane biosynthesis and platelet function in type II diabetes mellitus. $\mathbf{N}$ Engl J Med.1990; 322:1769-1774.CrossrefMedlineGoogle Scholar

- 5Davì G, Averna M, Catalano I, Barbagallo C, Ganci A, Notarbartolo A, Ciabattoni G, Patrono C. Increased thromboxane biosynthesis in type lla hypercholesterolemia. Circulation.1992; 85:1792-1798.CrossrefMedlineGoogle Scholar

- 6Di Minno G, Davì G, Margaglione M, Cirillo F, Grandone E, Ciabattoni G, Catalano I, Strisciuglio $\mathrm{P}$, Andria G, Patrono C, Mancini M. Abnormally high thromboxane biosynthesis 
in homozygous homocystinuria: evidence for platelet involvement and probucol-sensitive mechanism. J Clin Invest.1993; 92:1400-1406.CrossrefMedlineGoogle Scholar

- 7Patrono C, Davì G, Ciabattoni G. Thromboxane biosynthesis and metabolism in relation to cardiovascular risk factors. Trends Cardiovasc Med.1992; 2:15-

20.CrossrefMedlineGoogle Scholar

- 8 Criqui MH, Coughlin SS, Fronek A. Noninvasive diagnosed peripheral arterial disease as a predictor of mortality: results from a prospective study. Circulation.1985; 4:768773. Google Scholar

- 9 Criqui MH, Langer RD, Fronek A, Feigelson HS, Klauber MR, McCann TJ, Browner D. Mortality over a period of 10 years in patients with peripheral arterial disease. $\mathbf{N}$ Engl J Med.1992; 326:381-386.CrossrefMedlineGoogle Scholar

- 10Coffmann JD. Intermittent claudication: be conservative. N Engl J Med.1991; 325:577578.CrossrefMedlineGoogle Scholar

- 11Criqui MH, Browner D, Fronek A, Klauber MR, Barrett-Connor E, Coughlin SS, Gabriel S. Peripheral arterial disease in large vessels is epidemiologically distinct from small vessel disease: an analysis of risk factors. Am J Epidemiol.1989; 129:11101119. CrossrefMedlineGoogle Scholar

- 12Violi F, Criqui M, Longoni A, Castiglioni C, and the ADEP Group. Relation between risk factors and cardiovascular complications in patients with peripheral vascular disease: results from the ADEP Study. Atherosclerosis.1996; 120:25-35. CrossrefMedlineGoogle $\underline{\text { Scholar }}$

- 13FitzGerald GA. Mechanisms of platelet activation: thromboxane $A_{2}$ as an amplifying signal for other agonists. Am J Cardiol.1991; 68:11B-15B.CrossrefMedlineGoogle Scholar

- 14Rose GA. The diagnosis of ischemic heart pain and intermittent claudication in field surveys. Bull World Health Organ.1962; 27:117-126. Google Scholar

- 15Fronek A. NoninvasiveDiagnostics inArterialDisease. New York, NY: McGraw-Hill; 1989:88-94.Google Scholar 
- 16Newman AB, Siscovick DS, Manolio TA, Polak J, Fried LP, Borhani NO, Wolfson SK, for the Cardioarterial Health Study (CHS) Collaborative Research Group. Ankle-arm index as a marker of atherosclerosis in the Cardioarterial Health Study. Circulation.1993; 88:837845.CrossrefMedlineGoogle Scholar

- 17McKenna M, Wolfson S, Kuller L. The ratio of ankle and arm arterial pressure as an independent predictor of mortality. Atherosclerosis.1991; 87:119128. CrossrefMedlineGoogle Scholar

- 18Dormandy JA, Murray GD. The fate of the claudicant: a prospective study of 1969 claudicants. Eur J Vasc Surg.1991; 5:131-133. CrossrefMedlineGoogle Scholar

- 19National Diabetes Data Group. Classification and diagnosis of diabetes mellitus and other categories of glucose intolerance. Diabetes.1979; 28:10391057. CrossrefMedlineGoogle Scholar

- 20WHO memorandum. Classification of hyperlipidemias and hyperlipoproteinemias. Circulation.1972; 45:501-508.CrossrefGoogle Scholar

- 21The fifth report of the Joint National Committee on Detection, Evaluation, and Treatment of High Blood Pressure (JNC V). Arch Intern Med.1993; 153:154183.CrossrefMedlineGoogle Scholar

- 22Roberts LJ II, Sweetman BJ, Oates JA. Metabolism of thromboxane $B_{2}$ in man: identification of twenty urinary metabolites. J Biol Chem.1981; 256:83848393. MedlineGoogle Scholar

- 23Ciabattoni G, Maclouf J, Catella F, FitzGerald GA, Patrono C. Radioimmunoassay of 11-dehydro-TXB ${ }_{2}$ in human plasma and urine. Biochim Biophys Acta.1987; 918:29-

\section{Google Scholar}

- 24Patrono C, Ciabattoni G, Remuzzi G, Gotti E, Bombardieri S, Di Munno O, Tartarelli G, Cinotti GA, Simonetti BM, Pierucci A. Functional significance of renal prostacyclin and thromboxane $A_{2}$ production in patients with systemic lupus erythematosus. J Clin Invest.1985; 76:1011-1018. CrossrefMedlineGoogle Scholar 
- 25Friedewald WT, Levy RI, Fredrickson DS. Estimation of the concentration of low-density lipoprotein cholesterol in plasma, without use of the preparative ultracentrifuge. Clin Chem.1972; 18:499-502. MedlineGoogle Scholar

- 26Notarbartolo A, Davì G, Averna M, Barbagallo MB, Ganci A, Giammarresi C, La Placa $\mathrm{FP}$, Patrono $\mathrm{C}$. Inhibition of thromboxane biosynthesis and platelet function by simvastatin in type Ila hypercholesterolemia. Arterioscler Thromb.1995; 15:247-251.LinkGoogle $\underline{\text { Scholar }}$

- 27Siegel S. Nonparametric Statistics for the Behavioral Sciences. New York, NY: McGraw Hill; 1956:184.Google Scholar

- 28Patrignani P, Filabozzi P, Patrono C. Selective cumulative inhibition of platelet thromboxane production by low-dose aspirin in healthy subjects. J Clin Invest.1982; 69:1366-1372. CrossrefMedlineGoogle Scholar

- 29Ciabattoni G, Pugliese F, Davì G, Pierucci A, Simonetti BM, Patrono C. Fractional conversion of thromboxane $\mathrm{B}_{2}$ to urinary 11-dehydro- $\mathrm{TXB}_{2}$ in man. Biochim Biophys Acta.1989; 992:66-70.CrossrefMedlineGoogle Scholar

- 30FitzGerald GA, Pedersen AK, Patrono C. Analysis of prostacyclin and thromboxane biosynthesis in cardiovascular disease. Circulation.1983; 67:1174-

\section{CrossrefMedlineGoogle Scholar}

- 31Ogren M, Hedblad B, Isacsson O, Janzon L, Jungquist G, Lindell SE. Non-invasively detected carotid stenosis and ischaemic heart disease in men with leg arteriosclerosis. Lancet.1993; 342:1138-1141.CrossrefMedlineGoogle Scholar

- 32Fitzgerald DJ, Roy L, Catella F, FitzGerald GA. Platelet activation in unstable coronary disease. N Engl J Med.1986; 315:983-989.CrossrefMedlineGoogle Scholar

- 33Rangemark C, Ciabattoni G, Wennmalm A. Excretion of thromboxane metabolites in healthy women after cessation of smoking. Arterioscler Thromb.1993; 13:777782. CrossrefMedlineGoogle Scholar 
- 34Minuz P, Barrow SE, Cockroft JR, Ritter JM. Prostacyclin and thromboxane biosynthesis in mild essential hypertension. Hypertension.1990; 15:469-474.LinkGoogle $\underline{\text { Scholar }}$

- 35Catella F, FitzGerald GA. Paired analysis of urinary thromboxane metabolites in humans. Thromb Res.1987; 47:647-656.CrossrefMedlineGoogle Scholar

- 36Vejar M, Fragasso G, Hackett D, Lipkin DP, Maseri A, Born GVR, Ciabattoni G, Patrono C. Dissociation of platelet activation and spontaneous myocardial ischemia in unstable angina. Thromb Haemost.1990; 63:163-168.CrossrefMedlineGoogle Scholar

- 37Knapp HR, Healy C, Lawson J, FitzGerald GA. Effects of low-dose aspirin on endogenous eicosanoid formation in normal and atherosclerotic men. Thromb Res.1988; 50:377-386.CrossrefMedlineGoogle Scholar

- 38Carlsson I, Benthin G, Petersson A, Wennmalm A. Differential inhibition of thromboxane $\mathrm{A}_{2}$ and prostacyclin synthesis by low dose acetylsalicylic acid in atherosclerotic patients. Thromb Res.1990; 57:437-444.CrossrefMedlineGoogle Scholar

- 39Reilly IAG, Doran JB, Smith B, FitzGerald GA. Increased thromboxane biosynthesis in a human preparation of platelet activation: biochemical and functional consequences of selective inhibition of thromboxane synthase. Circulation.1986; 73:13001309.CrossrefMedlineGoogle Scholar

- 40Ejim OS, Powling MJ, Dandona P, Kernoff PBA, Goodall AH. A flow cytometric analysis of fibronectin binding to platelets from patients with peripheral arterial disease. Thromb Res.1990; 58:519-524.CrossrefMedlineGoogle Scholar

- 41Devine DV, Anderstad G, Nugent D, Carter CJ. Platelet-associated factor XIII as a marker of platelet activation in patients with peripheral arterial disease. Arterioscler Thromb.1993; 13:857-862.CrossrefMedlineGoogle Scholar

- 42Sinzinger H, Virgolini I, Fitscha P. Platelet kinetics in patients with atherosclerosis. Thromb Res.1990; 57:507-516.CrossrefMedlineGoogle Scholar 
- 43Cella G, Zahavi J, de Haas HA, Kakkar VV. $\beta$-Thromboglobulin, platelet production time and platelet function in arterial disease. Br J Haematol.1979; 43:127-

136.CrossrefMedlineGoogle Scholar

- 44Baele G, Bogaerts H, Clements DL, Pannier R, Barbier F. Platelet activation during treadmill exercise in patients with chronic peripheral arterial disease. Thromb

Res.1981; 23:215-223.CrossrefMedlineGoogle Scholar

- 45Verstraete M. Platelet activation in patients with atherosclerosis of the arteries of the limbs. In: Vanhoutte PM, ed. Serotonin and the Cardioarterial System. New York, NY: Raven Press; 1985:171-177.Google Scholar

- 46FitzGerald GA, Smith B, Pedersen AK, Brash AR. Increased prostacyclin biosynthesis in patients with severe atherosclerosis and platelet activation. $\mathbf{N}$ Engl $\mathbf{J}$

Med.1984; 310:1065-1068.CrossrefMedlineGoogle Scholar

- 47Kaplan KL, Owen J. Plasma levels of $\beta$-thromboglobulin and platelet factor 4 as indices of platelet activation in vivo. Blood.1981; 57:199-202.CrossrefMedlineGoogle Scholar

- 48Antiplatelet Trialists' Collaboration. Collaborative overview of randomised trials of antiplatelet therapy, I: prevention of death, myocardial infarction, and stroke by prolonged antiplatelet therapy in various categories of patients. Br Med J.1994; 308:81106. CrossrefMedlineGoogle Scholar

- 49Balsano F, Violi F. Effect of picotamide on the clinical progression of peripheral vascular disease: a double-blind placebo-controlled study — the ADEP Group. Circulation.1993; 87:1563-1569.CrossrefMedlineGoogle Scholar

- 50Patrono C. Aspirin as an antiplatelet drug. N Engl J Med.1994; 330:12871294.CrossrefMedlineGoogle Scholar

\section{Previous}

